\title{
Profundidad del surco gingival en dientes primarios restaurados con coronas de acero cromo
}

\section{Resumen}

Objetivo: Evaluar la relación entre el ajuste marginal y la profundidad del surco gingival en dientes primarios restaurados con coronas de acero cromo. Métodos: Se midió la profundidad del surco gingival de 114 dientes primarios restaurados con coronas de acero cromo en 67 niños, ambos sexos, de 3 a 6 años de edad de la clínica de Estomatología Pediátrica de la UATx, México. La población en estudio fue dividida en tres grupos: a) experimental $(n=30), b)$ control $(\mathrm{n}=8)$, y c) control negativo $(\mathrm{n}=29)$. Previo consentimiento informado se tomaron los índices de placa (Löe- Silness 1967) e índice gingival en seis superficies; se midió el surco gingival con sonda periodontal y las coronas de acero cromo fueron evaluadas clínica y radiográficamente según su ajuste marginal y longitud cervical. Resultados: Los índices de placa y gingival no
Rubén Muñoz Delgado

Ana Margot Noriega Cerón ${ }^{2}$

Jesús Ortega Rocha mostraron diferencias significativas para los grupos experimental y control negativo. El 34\% de las coronas mostraron buen ajuste marginal, $33 \%$ un mal ajuste, $10.5 \%$ cortas, $7.9 \%$ largas y el $14 \%$ otro tipo de mal ajuste. La profundidad del surco gingival fue estadísticamente significativa para el primer y segundo molar inferior derechos y en los caninos inferiores ( $p>0.05$ ); la superficie disto-vestibular presentó la mayor profundidad del surco gingival $(2.34 \mathrm{~mm})$, siendo estadísticamente significativa ( $p>0.05)$. Conclusiones: La profundidad del surco gingival fue mayor en los dientes rehabilitados con coronas de acero cromo mal ajustadas y sobre contorneadas (largas). La presencia de placa bacteriana y gingivitis estuvo relacionada con las coronas de acero cromo mal ajustadas.

Palabras clave: coronas acero cromo, gingivitis, surco gingival, adaptación marginal.

Artigo Original

\section{Profundidade do surco gingival em dentes primarios restaurados com coroas de aço cromo}

\section{Resumo}

Objetivo: Avaliar a relação entre o ajuste marginal e a profundidade do sulco gengival em dentes decíduos restaurados com coroas de aço-cromo. Métodos: Mediu-se a profundidade do sulco gengival de 114 dentes decíduos

\footnotetext{
${ }^{1}$ Profesor Titular de la Especialidad en Estomatología Pediátrica, Facultad de Odontología de la Universidad Autónoma de Tlaxcala.

${ }^{2}$ Alumna egresada de la Especialidad en Estomatología Pediátrica de la Universidad Autónoma de Tlaxcala.

${ }^{3}$ Profesor de la Especialidad en Estomatología Pediátrica, Facultad de Odontología de la UATx.
} 
restaurados com coroas de aço- cromo em 67 crianças, ambos sexos, de 3 a 6 anos de idade da clínica de Estomatología Pediátrica da UATx, México. A população em estudo foi dividida em três grupos: a) experimental $(n=30), b)$ controle $(\mathrm{n}=8)$, e c) controle negativo $(\mathrm{n}=29)$. Após consentimento informado, avaliaram-se os índices de placa (Löe-Silness 1967) e índice gengival em seis superfícies; mediu-se o sulco gengival com sonda periodontal e as coroas de aço-cromo foram avaliadas clínica e radiograficamente, segundo ajuste marginal e comprimento cervical. Resultados: Os índices de placa e gengival não mostraram diferenças significativas para os grupos experimental e controle negativo. $34 \%$ das coroas mostraram ajuste marginal adequado,
$33 \%$ ajuste cervical insatisfatório, $10.5 \%$ estavam curtas, $7.9 \%$ longas e o $14 \%$ outro tipo de desajuste. A profundidade do sulco gengival foi estatisticamente significativa para o primeiro e segundo molar inferior direitos e nos caninos inferiores ( $p>0.05$ ); a superfície disto-vestibular apresentou a maior profundidade de sulco gingival $(2,34 \mathrm{~mm})$, sendo estatisticamente significativa ( $p>0.05$ ). Conclusões: A profundidade do sulco gengival foi maior nos dentes reabilitados com coroas de aço- cromo mau ajustadas e com sobrecontorno (longas). A presença de placa bacteriana e gingivite esteve relacionada com as coroas de aço-cromo mal ajustadas.

Palavras-chave: coroa aço-cromo, gingivite, sulco gingival, adaptação marginal.

\section{Original article}

\section{Depth of gingival sulcus in primary teeth restored with stainless steel crowns}

\section{Summary}

Purpose: To evaluate the relation between marginal adaptation and depth of gingival sulcus on primary teeth restored with stainless steel crowns. Methods: Depth of gingival sulcus was measured in 114 primary teeth restored with stainless steel crowns on 67 children, both sex, 3 to 6 years old in a Pediatric Dentistry Clinic at the Universidad Autónoma de Tlaxcala, México. The children were divided in three groups: a) experimental $(n=30)$, b) control $(n=8)$ and c) negative control $(n=29)$. Informed consent was obtained and plaque and gingival indexes (Löe and Silness 1967) were taken on six dental surfaces, depth of gingival sulcus was measured with a dental probe and stainless steel crowns were clinically and radiographically evaluated on its marginal adaptation and cervical length. Results: Plaque index and Gingival index were not statistically significant for experimental and negative control groups. $34 \%$ of stainless steel crowns showed good marginal adaptation, $33 \%$ poor adaptation, $10.5 \%$ were short and $7.9 \%$ long, $14 \%$ other kind of poor adaptation. Depth of Gingival sulcus was statistically significant on lower right first and second primary molars and for lower cuspids ( $p>0,05)$; disto-buccal surface showed the deepest measured of gingival sulcus $(2.34 \mathrm{~mm})$, wich was statistically significant ( $p>0,05)$. Conclusions: Depth of gingival sulcus was greater on primary teeth restored with long stainless steel crowns and poor marginal adaptation. Plaque presence and gingivitis were related with poor adapted stainless steel crowns. 
Keywords: stainless steel crowns, gingivitis, gingival sulcus, marginal adaptation.

\section{Introducción}

El surco gingival es el espacio virtual entre la cara interna de la encía marginal y el diente y forma parte de la unidad biológico-funcional del periodonto. El periodonto sano tiene un surco gingival poco profundo que varía de $1.5 \mathrm{~mm}$ a $3 \mathrm{~mm}$ en la dentición permanente. ${ }^{1,2}$ Por su parte, en la dentición temporal aun no está claramente establecido dicho valor debido a que está sometido a diversos cambios fisiológicos y biológicos que pueden influir en su disposición anatómica. ${ }^{3}$ Uno de los principales problemas en la comprensión de la patogenia de la enfermedad periodontal en la infancia es la dificultad para diferenciar con claridad las condiciones normales de las patológicas. El sondeo es un procedimiento sencillo de diagnóstico muy útil que puede ayudar a identificar prematuramente la presencia de la inflamación gingival; omitirlo puede ocasionar que se pasen desapercibidos estados patológicos más severos con complicaciones sistémicas posteriores.

Hasta el momento no se conoce con exactitud la medida del surco gingival en niños, esto condiciona que comúnmente se lesione el epitelio de unión al realizar restauraciones mal ajustadas en el área cervical e interproximal, coronas pre-formadas e incluso aparatología de ortodoncia. ${ }^{4,5}$ La profundidad promedio del surco gingival en la dentición primaria es de $2.1 \mathrm{~mm}$ (+ $0,2)$ y que durante la erupción dental este puede profundizarse de 5 a $6 \mathrm{~mm}$ sin presentar sangrado (Nahas Correa 2005). ${ }^{6}$

En la fase de dentición mixta la migración del epitelio de unión se desarrolla a consecuencia de los procesos de exfoliación de los dientes tempo- rales y la erupción de los dientes permanentes (Eber y col. 2002). Es una estructura en renovación continua (Litsgarden 1966) por lo que establecer una medida estándar en esta etapa resulta prácticamente imposible. ${ }^{78,9,10}$ La profundidad del surco en la dentición permanente varía entre 1 a $2 \mathrm{~mm}$ dependiendo de la zona del diente a evaluar, por vestibular el promedio es de $1.15 \mathrm{~mm}, 2.05 \mathrm{~mm}(+0,5)$ en áreas proximales. ${ }^{11,12}$

Las coronas pre-formadas de acero cromo forman parte del inventario de la odontología restauradora en dientes temporales con varias superficies lesionadas. Su alta resistencia y durabilidad las hacen una restauración de primera elección. Están compuestas por un 70\% de hierro y del 9 a $12 \%$ de níquel; son blandas y maleables lo que facilita su recorte y adaptación. ${ }^{13} \operatorname{Sin}$ embargo, la reacción de los tejidos gingivales adyacentes inicia desde su manipulación mecánica; cuando los contornos son mal definidos y pobremente ajustados, pueden ser causa de inflamación en la zona del surco gingival y encía interdental. ${ }^{14,15}$

El objetivo de este estudio fue evaluar la relación entre el ajuste marginal y la profundidad del surco gingival en dientes temporales restaurados con coronas de acero cromo.

\section{Materiales y métodos}

El estudio consistió en la selección de 150 pacientes pediátricos sanos, ambos sexos, entre 3 y 6 años de edad asistentes a la clínica de estomatología pediátrica de la Universidad Autónoma de Tlaxcala que fueron rehabilitados con coronas de acero cromo durante el periodo de Enero del 2011 a Marzo del 2013. De esta población se seleccionó una muestra de 67 niños de ambos sexos que cumplían con los criterios de inclusión. A cada paciente se le explico la naturaleza 
y objetivos del estudio y previo consentimiento informado se procedió a la evaluación clínica.

Los sujetos fueron divididos en tres grupos: a) grupo experimental $(n=30)$, b) grupo control $(n=8)$ y c) grupo control negativo $(n=29)$. A cada paciente se le realizó la historia clínica de rutina, se obtuvo el índice de placa bacteriana de Löe- Silness (Löe 1967), valorando las superficies mesial, distal, vestibular y lingual con un espejo bucal y explorador. Posteriormente se obtuvo el índice gingival (Löe-Silnees 1967) mediante la inspección visual de cada superficie y se midió la profundidad del surco gingival con

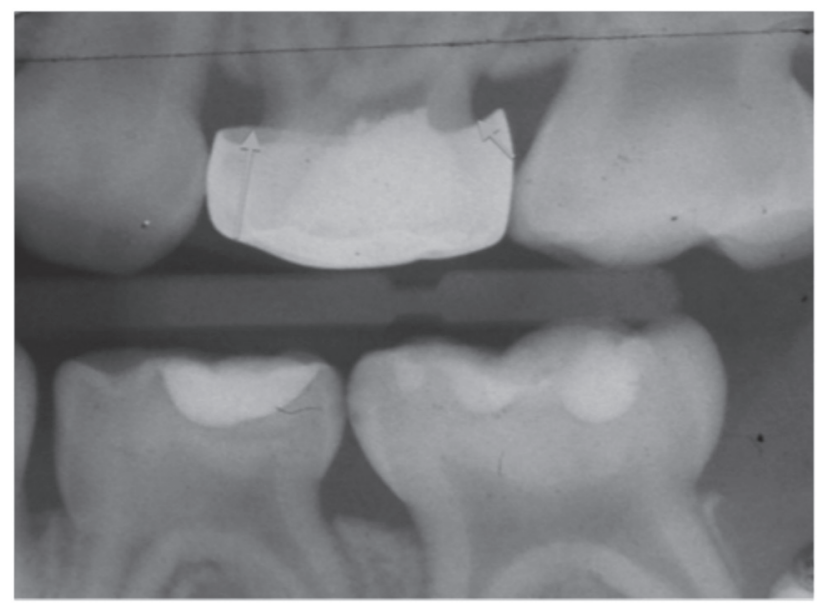

Figura 1. Evidencia radiográfica de un mal ajuste marginal de la corona de acero en la zona cervical (flechas).

\section{Indice de Placa (IP) e Indice Gingival (IG) por grupos}

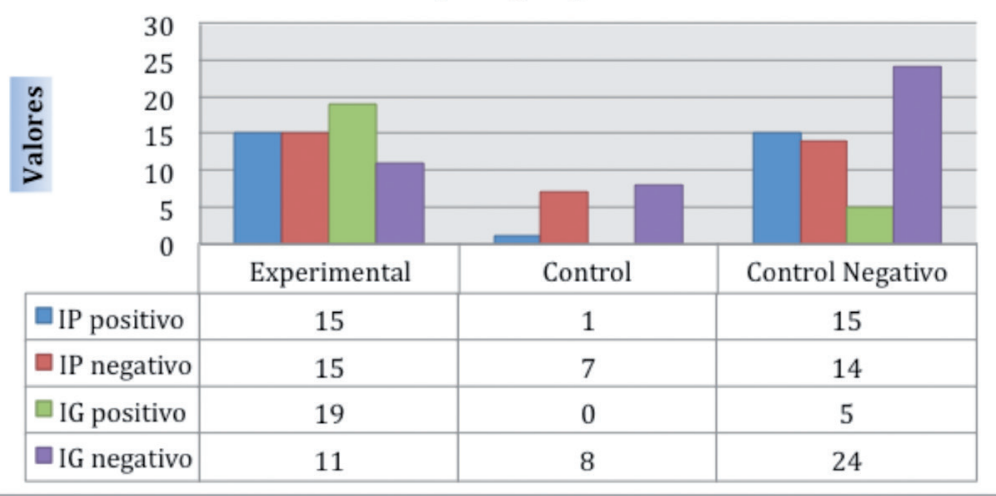

Figura 2. Resultados del Índice Gingival e Índice de Placa por grupos. sonda periodontal tipo Williams y espejo intraoral en cada una de las seis superficies del diente (Figura 1). Como último paso se tomó una radiografía con un colimador de aleta de mordida (Rinn Xcp Denstply) para evaluar radiográficamente el ajuste las coronas de acero cromo mediante una escala simple. (Tabla 1)

Los datos obtenidos fueron analizados y organizados mediante estadística descriptiva y aritmética. Se emplearon la prueba de $\mathrm{t}$ student $\mathrm{y}$ prueba $\mathrm{Z}$ para diferencia de medias en muestras pequeñas y grandes respectivamente.

Tabla 1. Criterios de evaluación de las coronas

\begin{tabular}{ccc}
\hline Código & Tipo & Definición \\
\hline A & Cortas & El margen de la corona está 2mm o más por arriba de la \\
constricción cervical.
\end{tabular}




\section{Resultados}

El $61 \%(n=41)$ de los sujetos fueron del sexo masculino y $39 \%$ ( $n=26)$ del sexo femenino; el promedio de edad fue de 4.68 años (ds=1.05). El 50\% de los sujetos del grupo experimental dieron positivo al índice de placa (IP) y el 50\% negativo. En el grupo control negativo, el 52\% dio positivo al IP contra un $48 \%$ negativo. Las diferencias no fueron estadísticamente significativas. En el grupo control el $87.5 \%$ dio negativo para el IP. En el índice gingival (IG) el 63\% del grupo experimental dio positivo y un $37 \%$ negativo; en el grupo control negativo el $17 \%$ fue positivo contra un $83 \%$ negativo. En el grupo control el $100 \%$ de los sujetos dieron negativo al IG (Figura 2).

De las 114 coronas evaluadas el 34\% mostraron buen ajuste marginal, 33\% un mal ajuste, 10.5\% cortas, $7.9 \%$ largas y un $14 \%$ mostraron algún otro tipo de mal ajuste (Tabla 2).

La profundidad del surco gingival mostró diferencias significativas para el segundo molar in- ferior derecho $(\mathrm{exp} .=2.01 \mathrm{ds}=0.73 \mathrm{vs}$. cont.$=1.39$ $\mathrm{ds}=0.75)$, los primeros molares inferiores (exp.= $1.88-2.0 \mathrm{ds}=0.8$ vs. cont. $=1,16 \mathrm{ds}=0,7)$, caninos inferiores $(\exp .=1.83 \mathrm{ds}=0.9 \mathrm{vs}$. cont $.=1.04 \mathrm{ds}=$ $0.6)$, e incisivos central y lateral superior derechos $($ exp. $=1.83-95 \mathrm{ds}=0.8$ vs. contr. $=1.02 \mathrm{ds}=0.5)(\mathrm{p}$ $>0.05$ ) (Tabla 3). La medida del surco gingival por superficie mostró que la superficie disto-vestibular presentó la mayor profundidad del surco gingival (experimental $=2,34 \mathrm{ds}=0.69$ vs. control $=1.35 \mathrm{ds}=0.58)$ siendo estos valores estadísticamente significativos ( $\mathrm{p}>0.05)$ (Tabla 4 ).

\begin{tabular}{|c|c|c|c|}
\hline Grupo & Criterio & No. & $\%$ \\
\hline$A$ & Cortas & 12 & 10.52 \\
\hline B & Largas & 9 & 7.9 \\
\hline C & $\begin{array}{c}\text { Mal ajuste } \\
\text { marginal }\end{array}$ & 38 & 33.33 \\
\hline$D$ & $\begin{array}{c}\text { Adecuado ajuste } \\
\text { marginal }\end{array}$ & 39 & 34.21 \\
\hline E & Otras & 16 & 14.03 \\
\hline Total & & 114 & $100 \%$ \\
\hline
\end{tabular}

Tabla 3. Diferencias de las medias aritméticas de la profundidad del surco gingival por órgano dental.

\begin{tabular}{lc}
\hline \multicolumn{1}{c}{ Órgano Dental } & Número \\
\hline Segundo Molar & $55^{\mathrm{a}}, 65^{\mathrm{b}}, 75^{\mathrm{c}}, 85^{\mathrm{d}}$ \\
Primer Molar Superior Derecho & 54 \\
Primer Molar & $64^{\mathrm{a}}, 74^{\mathrm{b}}, 84^{\mathrm{c}}$ \\
Canino Superior Derecho & 53 \\
Canino Primario & $63^{\mathrm{a}}, 73^{\mathrm{b}}, 83^{\mathrm{c}}$ \\
Incisivos Lateral Primario & $52^{\mathrm{a}}, 62^{\mathrm{b}}, 72^{\mathrm{c}}$ \\
Incisivo Lateral Inferior Derecho & $82^{2}$ \\
Incisivo Central Superior e Inferior Derechos & $51^{\mathrm{a}}, 71^{\mathrm{b}}$ \\
Incisivo Central Superior e Inferior Izquierdos & $61^{\mathrm{a}}, 81^{\mathrm{b}}$ \\
\hline
\end{tabular}

Letras diferentes representan diferencias significativas con $t$ student y un valor de $\mathrm{p}<0.05$. 
Tabla 4. Profundidad promedio del surco gingival por superficie

\begin{tabular}{ccc} 
Superficie & $E$ & $C$ \\
\hline DV & $2.34 \pm 0.69^{\mathrm{a}}$ & $1.35 \pm 0.58^{\mathrm{b}}$ \\
V & $1.02 \pm 0.66^{\mathrm{a}}$ & $0.65 \pm 0.47^{\mathrm{b}}$ \\
MV & $2.12 \pm 0.76^{\mathrm{a}}$ & $1.36 \pm 0.61^{\mathrm{b}}$ \\
DP/ DL & $2.15 \pm 0.72^{\mathrm{a}}$ & $1.36 \pm 0.62^{\mathrm{b}}$ \\
P/ L & $1.07 \pm 0.7^{\mathrm{a}}$ & $0.87 \pm 0.41^{\mathrm{b}}$ \\
MP / ML & $1.9 \pm 0.7^{\mathrm{a}}$ & $1.26 \pm 0.61^{\mathrm{b}}$ \\
\hline
\end{tabular}

E, grupo experimental; C, grupo control; DV, distovestibular; V, vestibular, MV, mesiovestibular; DP/DL, distopalatino/lingual; $\mathrm{P} / \mathrm{L}$, palatino/lingual; MP/ML, mesiopalatino/lingual; Letras diferentes representan diferencias significativas con $p<0.05$

\section{Discusión}

Conocer las características normales del periodonto en la dentición infantil es importante para poder distinguir entre la salud y enfermedad y de esta manera realizar un diagnóstico correcto de las enfermedades periodontales. Durante la niñez y la pubertad, el periodonto está en constante cambio debido a la exfoliación y erupción de los dientes lo cual hace difícil la descripción de un periodonto normal (Baer y col 1975, Bimstein y col 1999). ${ }^{16,17}$

El rango de edad de los sujetos de este estudio fue de 3 a 6 años, periodo durante el cual el proceso de erupción esta temporalmente detenido y los cambios en el periodonto infantil suelen ser mínimos. A partir de los 5 años, el periodonto sufre cambios localizados con la exfoliación de los incisivos centrales inferiores y la erupción de los primeros molares permanentes (Bimstein 2001). En relación al género no se observaron diferencias relevantes del periodonto infantil.

Considerando que el factor principal en la etiología de la enfermedad periodontal, es la presencia y acumulación de placa bacteriana ${ }^{18}$ el Índice de Placa bacteriana (IP) de Löe- Silness se considero como un instrumento útil para la evaluación clínica; observándose que en el grupo experimental no hubo diferencias entre los sujetos, mientras que en el grupo control la diferencia fue considerable (7:1). Esto confirma que la higiene bucal en una persona es el factor más importante para la salud gingival más que la mera presencia de una restauración, la cual bajo ciertas condiciones puede alterar el estado de los tejidos gingivales. (Checcio 1983) ${ }^{19}$

La gingivitis inducida por placa bacteriana depende de la etapa de formación, organización y maduración; así como del desarrollo del sistema inmune y endocrino. ${ }^{17,20}$ La gingivitis en el niño no es tan severa como en el adulto debido a quela flora bacteriana de los niños es menos patógena (Bimstein et al, 1999). La tendencia a desarrollar gingivitis en niños y adolescentes puede estar asociada a alteraciones en la composición microbiana de la placa dental, la respuesta inflamatoria celular, cambios hormonales, diferencias morfológicas, exfoliación y erupción de los dientes (Bimstein y Mattson, 1999, Jenkin $2001)^{21,22,23}$.

El índice de gingivitis mostro diferencias importantes con el grupo control negativo (19:5) debido a que, los órganos adyacentes en el mismo sujeto no presentaron gingivitis; asumiendo que 
ésta, estuvo relacionada con la presencia de coronas de acero cromo. Mientras que los sujetos del grupo control no mostraron gingivitis. Podemos deducir que la presencia de la corona de acero cromo es un factor condicionante para el desarrollo de la inflamación gingival. Checcio y cols. en 1983, reportaron que la inflamación inicial se debe a la irritación local después de la colocación de una corona y que esta puede aumentar si la higiene oral no es la adecuada que impida mantener un área limpia alrededor de ellas $^{19}$. Durr y cols. en 1982, postularon que la placa subgingival no detectada puede acumularse en el espacio entre los márgenes de la corona y el diente y así contribuye a provocar una inflamación gingival. ${ }^{24}$

Por el contrario, Machen y cols. en 1980 no encontraron diferencias significativas en los tejidos gingivales que rodean los dientes restaurados y el tejido circundante en órganos sin coronas. ${ }^{25}$ Richter y Ueno en 1973, no encontraron relación de los tejidos gingivales con los márgenes de las coronas. $^{26}$

Padilla y $\operatorname{cols}^{27}$ en 2007 realizaron la medición de surco gingival en dientes temporales en un grupo de 100 niños de Tlaxcala entre 4 y 5 años de edad, clínicamente sanos. Encontraron que la profundidad del surco gingival promedio de las 6 caras fue de $1 \mathrm{~mm}$ a excepción de los segundos molares superiores que fue de $2 \mathrm{~mm}$. Estos valores son similares con los datos obtenidos en nuestro estudio cuyo rango fue de 1 a $1.47 \mathrm{~mm}$ en la mayoría de los dientes sanos y de 1.30 a 1.39 para los segundos molares superiores.

El correcto ajuste de las coronas preformadas es fundamental para evitar la irritación mecánica de los tejidos gingivales, ya que un mal ajuste predispone a una severa inflamación gingival. (Henderson 1973, Myers 1980, Checcio 1983). ${ }^{28}$
En nuestro estudio el mal ajuste marginal fue el error más observado (33.3\%), refiriéndonos a este, cuando la corona de acero no fue correctamente "crimpeada" o "cinchada" a nivel cervical y sub-gingival (Fig. 1), lo que condiciono la presencia de inflamación gingival alrededor de los dientes involucrados. Palomo y Peden en 1976, señalaron que las coronas con extensión subgingivales (largas) producen mayor inflamación gingival que aquellas ajustadas por encima del margen gingival. ${ }^{29}$ El contorno inadecuado de los márgenes y los residuos de cemento en contacto con el surco gingival puede ocasionar una inflamación local del surco al tiempo que favorece la acumulación de placa bacteriana ${ }^{30}$.

Las coronas de acero cromo preformadas tienen una dimensión estándar y no siempre coinciden con la dimensión del diente natural. Si estas no son ajustadas correctamente se puede invadir el epitelio de unión y lesionarlo considerablemente. Este epitelio puede migrar y se reinserta más apicalmente permitiendo la acumulación de placa bacteriana y la inflamación subsecuente. ${ }^{30}$

El llamado "espesor biológico" corresponde a la distancia entre el limite amelo-cementario y la altura de la cresta ósea alveolar. En dientes primarios se ha propuesto que una distancia de 2 $\mathrm{mm}$, medidos radiográficamente, se considera como un espesor biológico normal y saludable. Sin embargo, es normal encontrar dimensiones mayores a $2 \mathrm{~mm}$ en dientes primarios próximos a exfoliar y en los dientes permanentes adyacentes en erupción. En niños con dentición mixta, en los cuales existen dientes en reciente erupción se pueden encontrar profundidades del surco gingival mayores a $3 \mathrm{~mm}$, lo cual no es un signo necesariamente de enfermedad periodontal (Baer y col, 1975). ${ }^{31}$

Los márgenes de la corona deben ser continuos sin esquinas, ángulos agudos, líneas rectas y sin 
bordes cortantes (Spedding 1984). Si se tiene presente la longitud de la corona y la forma de los márgenes, la adaptación de ésta será óptima y el resultado clínico satisfactorio. Recomendamos el uso del colimador durante la toma radiográfica para verificar el ajuste final de las coronas ya que nos da un mejor ángulo de la imagen y así poder asegurar que la longitud y adaptación cervical proximal sean correctas y evitar efectos negativos en los tejidos periodontales.

\section{Conclusiones}

La profundidad del surco gingival fue mayor en los dientes rehabilitados con coronas de acero cromo mal ajustadas y sobre contorneadas -largas- en la zona cervical. La presencia de placa bacteriana y gingivitis estuvo relacionada con las coronas de acero cromo mal ajustadas.

El correcto ajuste marginal de las coronas de acero cromo, si invadir o lesionar el surco gingival aseguran una buena salud gingival.

\section{Bibliografía}

1. Newman T. Periodontología Clínica de Carranza. 9a ed. McGraw-Hill Interamericana, 2010.

2. Ten Cate Ar. Histología Oral. Desarrollo, Estructura y Función, 2ª ed. Buenos Aires: Ed. Panamericana; 1986.

3. Koch G, Poulsen S. Odontopediatría: Abordaje Clínico, 2 ed. Editorial Amolca; 2011.

4. Cohen B: Morphological factors in the pathogenesis of periodontal disease. Br Dent J 1959; 107:31.

5. Baer P, Benjamin D. Enfermedades Periodontales en niños y adolescents. $1^{\mathrm{a}}$ ed. Buenos Aires: Editorial Mundi, 1975.

6. Nahas P Corréa MS. Odontopediatria Na Primera Infancia. $2^{a}$ ed; 2005.

7. Gómez de Ferraris M. Histología y Embriología Bucodental. $3^{\mathrm{a}}$ ed. Panamericana; 2009.

8. Andli-Sobocki A. Changes of facial gingival dimensions in children: A 2 year longitudinal study. J. Clin Periodontol 1993; 20: p. 212-218.

9. Gottlieb B, Orban B: Active and passive continuous eruption of teeth. J Dent Res 1933; 13:214.

10. Listgarten Ma: Phase-Contrast and electron microscopic study of the junction between reduced enamel epithelium and enamel in unerupted human teeth. Arch Oral Biol 1966; 11, 999.

11. Ainamo J. Löe H. Anatomical Characteristics Of Gingiva: A Clinical and Microscopic study of the free and attached gingival. J Periodontol 1966; 37:5.

12. Gargiulo Aw, Wentz Fm, Orban B: Dimensions and Relations of the Dentogingival Junction in Humans. J Periodontol $1961 ; 32: 261$.

13. Guedes Pinto A. Rehabilitación Bucal En Odontopediatría. 1a ed. Santa Fe De Bogotá: Editorial Amolca; $2007 ;$ p.233.

14. Periodontal Diseases of Children and Adolescent. Reference Manual Update Pediatr Dent 2006- 2007; p. 204-216.

15. Myers DR, et al. The effect of polishing techniques on surface smoothness and plaque accumulation on stainless Steel crowns. Update Pediatr Dent 1980; 2:275-78.

16. Bimstein E. Matsson L. Growth and development considerations in the diagnosis of gingivitis and periodontitis in children. Update Pediatr Dent 1999; 213. 186-191.

17. Schroeder He, Listgarten Ma: Fine structure of the developing epithelial attachment of human teeth. Monographs in Developmental Biology Basel Karger 1971 Vol. 2.

18. Marsh Pd, Dental Plaque: Biological Significance of a Biofilm and Community Life-Style. J Clin Periodontol 2005; 32 (Supl 6): 7-15.

19. Checchio LM, Gaskill WF, Carrel R. The relationship between periodontal disease and stainless Steel crowns. J Dent Child 1983; 50: 205-9. 
20. Socransky Ss, Haffajee Ad: Dental Biofilms: Difficult Therapeutic Targets. Periodontol 2000 2002; 28:12-55.

21. Murrieta PF, Juárez LA. Prevalencia de Gingigivitis en un grupo de escolares y su relación con el grado de higiene oral y el nivel de conocimientos sobre salud bucal demostrado por sus madres. Bol. Med. Hosp. Infant Mex. 2004 ; Vol. 61:1 pp.44-54.

22. Jenkin W, Papapanou P. Epidemiology of periodontal diseases in children and adolescents. Periodontol 2000 2001, 26:16-32.

23. Kimura A, Ooshima T, Takiguchi M, Et Al: Periodontopathic Bacterial Infection in Childhood. J Periodontol 2002; 73: 20-6.

24. Durr DP, Ashrafi MH, Duncan WK. Astudy of plaque accumulation and gingival health surrounding stainless Steel crowns. J Dent Child 1982; 49:343- 6.

25. Machen DE, Rapp R, et al. The effect of Stainless Steel crowns on marginal gingival tissue. J Dent Res 1980; 59 (Abstract) p.239.

26. Richter W, Ueno H. Relationship of cast margin placement to gingival inflammation. J Prosthet Dent 1973; 3 0: 156-60.

27. Padilla M. Medida del surco gingival clínico de la dentición temporal en niños de 4 y 5 años de edad con tejidos periodontales clínicamente sanos de Tlaxcala [Tesis]. Universidad Autónoma de Tlaxcala: Facultad de Odontología 2011.

28. Henderson HZ. Evaluation of the preformed stainless Steel crowns. J Dent Child 1973; 40:353- 8.

29. Palomo F, Peden J. Periodontal considerations of restorative procedures. J Prosthet Dent 1976; 36:387-91.

30. Myers DR. A clinical study of the response of the gingival tissues surrounding stainless Steel crowns. J Dent Child 1975; 42: 28-34.

31. Pöllänen Mt, Salonen Jl Uitto Vj: Structure and Function of the Tooth- Epitelial Interface in Health and Disease. Periodontol 2000 2003;31:12.

Fecha de recepción 02/05/14

Fecha de aceptación 06/11/14 